\title{
On the vibrational spectrum of $\mathrm{C}_{9}, \mathrm{C}_{11}$ and $\mathrm{C}_{13}$
}

\author{
Jan M.L. Martin ${ }^{\mathrm{a}, \mathrm{b}}$, Peter R. Taylor $^{\mathrm{c}}$ \\ a Limburgs Universitair Centrum, Department SBG, Universitaire Campus, 3590 Diepenbeek, Belgium \\ b University of Aniwerp (UIA), Institute for Materials Science, Department of Chemistry, Universiteitsplein 1, 2610 Wilrijk, Belgium \\ c San Diego Supercomputer Center, P.O. Box 85608, San Diego, CA 92186-9784, USA
}

Received 23 March 1995; in final form 11 May 1995

\begin{abstract}
The harmonic frequencies and infrared intensities of $\mathrm{C}_{9}, \mathrm{C}_{11}$ and $\mathrm{C}_{13}$ have been calculated using SCF and complete active space SCF (CASSCF) methods. The ordering of the harmonic frequencies in $\mathrm{C}_{9}$ is predicted wrongly unless at least the $\pi$ HOMO and LUMO are included in the active space. Infrared intensities depend crucially on the size of the active space. For linear odd-numbered clusters $\mathrm{C}_{13}$ and larger, the computed SCF spectrum is qualitatively wrong. The recent observation of a band near $1809 \mathrm{~cm}^{-1}$ in the gas phase is explained using our CASSCF results on $\mathrm{C}_{13}$.
\end{abstract}

\section{Introduction}

Small carbon clusters have been the subject of many extensive theoretical and experimental studies (see Refs. [1,2] and the introduction to Ref. [3] for reviews), even before the development of fullerene chemistry. The diatomic $\mathrm{C}_{2}$ has been studied extensively [4], while the linear clusters $C_{n}(n=3-7,9)$ have been characterized by high-resolution infrared spectroscopy. Very recently, high-level ab initio frequency calculations [5] presented convincing evidence for the existence of cyclic $\mathrm{C}_{6}, \mathrm{C}_{8}$, and $\mathrm{C}_{10}$ in matrix infrared spectra of trapped carbon vapor [6,7], while the assignment of a band at $1284 \mathrm{~cm}^{-1}$ to cyclic $\mathrm{C}_{4}$ [8] was confirmed with virtual certainty in a large-basis set calculation of the quartic force field of $\mathrm{C}_{4}$ [9]. Further, accurate ab initio heats of formation are available for $\mathrm{C}_{3}$ through $\mathrm{C}_{10}$ inclusive [10].

Very little is known about clusters larger than $C_{10}$ but still below the fullerene regime in size, except that there appears to be a consensus in the literature that they are cyclic. However, very recently, a pa- per by Giesen et al. (denoted GOHFPS) [11] appeared which reports high-resolution infrared spectra for a band at 1808.96399 (7) $\mathrm{cm}^{-1}$ that, from the rotational fine structure, unambiguously belonged to a linear $\mathrm{C}_{13}$ molecule with average hond distance $r_{\text {eff }}=$ $1.2770(5) \AA$. The nearest band in the matrix infrared spectra $[6,7]$ is one at $1804 \mathrm{~cm}^{-1}$ : however, not only is the intensity variation upon annealing of this band strongly correlated with another band at $1844 \mathrm{~cm}^{-1}$ (no corresponding band to which is found by GOHFPS), but previous studies indicate [12] a linear relationship between cluster size and matrix red-shift, from which the observed matrix band of linear $\mathrm{C}_{13}$ would be predicted between 1780 and $1795 \mathrm{~cm}^{-1}$. No such band is found in the matrix IR spectra, which suggests that linear $\mathrm{C}_{13}$ is not present in the matrix experiments. Hence linear $\mathrm{C}_{13}$ is presumably thermodynamically metastable. GOHFPS explain that, at the high temperatures involved in laser vaporization, linear structures are strongly favored by entropy, while the fast cooling down in supersonic expansion may 
never give these high-energy structures the chance to relax to more stable cyclic isomers. Moreover, high barriers towards isomerization may exist, as demonstrated by Von Helden et al. [13,14] in their experimental and theoretical study on $\mathrm{C}_{7}$.

While Hartree-Fock level frequency calculations on the smaller clusters [15] had been instrumental in the experimental identification of several smaller species, such as $C_{4}[16], C_{5}[17], C_{6}[18,19], C_{7}[20]$ and $\mathrm{C}_{9}$ [21], SCF-level calculations by one of the authors [22] on $\mathrm{C}_{11}$ through $\mathrm{C}_{19}$ did not exhibit any calculated band for $\mathrm{C}_{13}$ that could plausibly correspond to the observations of GOHFPS. Second-order perturbation theory (MP2) calculations on the smaller clusters [23] yield results that differ increasingly from nature as the cluster size increases, with qualitatively incorrect results being predicted for $\mathrm{C}_{9}$. One plausible explanation might be that nondynamical correlation increases steadily with chain length: under such circumstances, a Hartree-Fock treatment might benefit from a cancellation of errors between neglect of dynamical and nondynamical correlation, whereas a low-order perturbation theory treatment will not benefit from such a cancellation since dynamical correlation is treated explicitly. However, such a cancellation of errors in Hartree-Fock must also eventually break down.

The present Letter reports attempts to perform more refined ab initio frequency calculations for $C_{11}$ and $\mathrm{C}_{13}$. Since extensive material is available for $\mathrm{C}_{9}[5]$, this molecule will be used as a gauge for the reliability of our calculations.

\section{Computational methods}

All calculations were performed using Dunning's correlation consistent polarized valence double zeta (cc-pVDZ) basis set [24], which is a [3s2p1d] contraction of a $(9 \mathrm{~s} 4 \mathrm{p} 1 \mathrm{~d})$ primitive set. Experience suggests $[25,26]$ that this basis set is generally much more reliable for correlated frequency calculations than the commonly used Huzinaga-Dunning [27,28] doublezeta plus polarization (DZP) basis set.

Because of the size of the largest system, $\mathrm{C}_{13}$, coupled-cluster frequency calculations, which would have been our approach of choice, are virtually out of the question even in this modest basis set. Since our main interest here is to treat nondynamical correlation adequately, the best alternative for a system this size is CASSCF (complete active space SCF) [29]. The calculations were carried out on the CRAY C90 computer at the San Diego Supercomputer Center using the SIRIUS/ABACUS [30] program system. Hartree-Fock level frequency calculations were carried out using TURBOMOLE [31] on an IBM RS/6000 model 350 workstation at SDSC. In both cases, analytical second derivatives were used, and infrared intensities were determined within the double-harmonic approximation. We may note that the cc-pVDZ basis set would not necessarily be expected to yield reliable IR intensities even at the SCF or CASSCF level without some augmentation with diffuse functions. However, the intensities computed here are used primarily for comparisons, and the $c c-p V D Z$ set is adequate for this purpose.

Active spaces for the CASSCF calculations were determined from natural orbital occupations at the MP2 level. The obvious candidate would be an active space consisting of the HOMO $\left(\pi_{\mathrm{g} / \mathrm{u}}\right)$ and LUMO $\left(\pi_{\mathrm{u} / \mathrm{g}}^{*}\right)$ orbitals, which leads to a 4-electron in 4orbital, or CASSCF (4/4), active space. However, the MP2 calculations suggested that the natural orbital occupations of $\pi \mathrm{HOMO}-1$ and LUMO +1 deviated significantly from their Hartree-Fock values of four and zero, respectively, as well, suggesting further calculations involving a $\operatorname{CASSCF}(8 / 8)$ wave function.

\section{Results and discussion}

Table 1 contains calculated results for $\mathrm{C}_{9}$, together with CCSD(T)/cc-pVDZ [5], MP2/6-31G* [23], density functional [3], and ad hoc MNDO [32] results from the literature.

We immediately see that all methods predict the same ordering of the stretching frequencies, except for HF/cc-pVDZ and MP2/6-31G*. At the HF/cc-pVDZ level, the second highest $\sigma_{\mathrm{g}}$ and $\sigma_{\mathrm{u}}$ frequencies are interchanged, while at the MP2/6-31G* level, the top $\sigma_{\mathrm{g}}$ and $\sigma_{\mathrm{u}}$ frequencies are interchanged. Of the results presented, it was shown amply in Ref. [5] that the CCSD(T)/cc-pVDZ ones should be the most reliable. It is striking how both the density functional results of Huitter et al. [3] and the MNDO (modified neglect of diatomic overlap [33]) calculations with parameters 
Table 1

Computed frequencies $\left(\mathrm{cm}^{-1}\right)$ and IR intensities $(\mathrm{km} / \mathrm{mol})$ for $\mathrm{C}_{9}$

\begin{tabular}{|c|c|c|c|c|c|c|c|c|c|c|c|c|c|}
\hline & \multicolumn{2}{|c|}{$\begin{array}{l}\text { SCF } \\
\text { /cc-pVDZ } \\
\text { this work }\end{array}$} & \multicolumn{2}{|c|}{$\begin{array}{l}\text { CASSCF( } 4 / 4) \\
\text { /cc-pVDZ } \\
\text { this work }\end{array}$} & \multicolumn{2}{|c|}{$\begin{array}{l}\text { CASSCF }(8 / 8) \\
\text { /cc-pVDZ } \\
\text { this work }\end{array}$} & \multirow{2}{*}{$\begin{array}{l}\operatorname{CCSD}(T) \\
\text { /cc-pVDZ } \\
\text { Ref. [5] } \\
\text { freq. }\end{array}$} & \multicolumn{2}{|c|}{$\begin{array}{l}\text { MP2/6-31G* } \\
\text { Ref. [23] }\end{array}$} & \multicolumn{2}{|c|}{$\begin{array}{l}\text { Density } \\
\text { functional } \\
\text { Ref. [3] }\end{array}$} & \multicolumn{2}{|c|}{$\begin{array}{l}\text { Ad hoc } \\
\text { MNDO } \\
\text { Ref. [32] }\end{array}$} \\
\hline & freq. & inten. & freq. & inten. & freq. & inten. & & freq. & inten. & freq. & inten. & freq. & inten. \\
\hline$\sigma_{\mathrm{g}}$ & 2419 & 0 & 2421 & 0 & 2340 & 0 & 2212 & 2287 & 0 & 2209 & 0 & 2266 & 0 \\
\hline$\sigma_{\mathrm{u}}$ & 2340 & 572 & 2267 & 589 & 2276 & 8014 & 2148 & 2426 & 2628 & 2182 & 2324 & 2196 & $\mathrm{RI}=4.4$ \\
\hline$\sigma_{\mathrm{u}}$ & 2087 & 41784 & 2165 & 28607 & 2174 & 2578 & 2061 & 2133 & 824 & 2073 & 3030 & 2042 & $\mathrm{RI}=22.9$ \\
\hline$\sigma_{\mathrm{g}}$ & 2131 & 0 & 2115 & 0 & 2093 & 0 & 1898 & 1930 & 0 & 1903 & 0 & 1938 & 0 \\
\hline$\sigma_{\mathrm{u}}$ & 1800 & 1050 & 1797 & 85 & 1775 & 780 & 1607 & 1635 & 190 & 1624 & 377 & 1676 & $\mathrm{RI}=1.5$ \\
\hline$\sigma_{\mathrm{g}}$ & 1390 & 0 & 1390 & 0 & 1382 & 0 & 1247 & 1267 & 0 & 1247 & 0 & 1312 & 0 \\
\hline$\sigma_{\mathbf{u}}$ & 958 & 32 & 955 & 33 & 960 & 4 & 864 & 879 & 0.4 & 905 & 12 & 905 & RI small \\
\hline$\pi_{\mathbf{u}}$ & 740 & 56 & 739 & 29 & 737 & 12 & & & & 496 & 111 & 657 & $\mathrm{RI}=0.1$ \\
\hline$\pi_{\mathrm{g}}$ & 657 & 0 & 652 & 0 & 651 & 0 & & & & 500 & 0 & 628 & 0 \\
\hline$\sigma_{\mathrm{g}}$ & 495 & 0 & 495 & 0 & 495 & 0 & 449 & 457 & 0 & 458 & 0 & 467 & 0 \\
\hline$\pi_{\mathrm{u}}$ & 568 & 1 & 546 & 4 & 551 & 0 & & & & 442 & 13 & 543 & RI small \\
\hline$\pi_{\mathrm{g}}$ & 495 & 0 & 290 & 0 & 335 & 0 & & & & 255 & 0 & 350 & 0 \\
\hline$\pi_{\mathrm{u}}$ & 206 & 3 & 210 & 2 & 253 & 8 & & & & 184 & 435 & 248 & RI small \\
\hline$\pi_{\mathrm{g}}$ & 121 & 0 & 129 & 0 & 143 & 0 & & & & 116 & 0 & 132 & 0 \\
\hline$\pi_{\mathrm{u}}$ & 49 & 5 & 54 & 6 & 55 & 6 & & & & 49 & 161 & 49 & RI small \\
\hline
\end{tabular}

RI denotes the relative intensity with respect to the $2039 \mathrm{~cm}^{-1}$ band of $C_{3}(R I=1.0)$.

specifically optimized for carbon clusters [32] are in very good agreement with the $\operatorname{CCSD}(\mathrm{T}) / \mathrm{cc}-\mathrm{pVDZ}$ values. Both levels of theory suggest two very intense bands in the $2000-2150 \mathrm{~cm}^{-1}$ range: the lower of the two bands is known to correspond to the $1998 \mathrm{~cm}^{-1}$ matrix IR feature which becomes extremely intense upon strong annealing, while it was shown in Ref. [5] that the upper one is almost certainly a component of a broad, convoluted, feature stretched out between 2080 and $2095 \mathrm{~cm}^{-1}$. In addition, the third lowest IR active band was assigned to features around $1600 \mathrm{~cm}^{-1}$ in Ref. [5].

At the SCF level, the band corresponding to the $1998 \mathrm{~cm}^{-1}$ matrix feature has an anomalously high intensity $(41784 \mathrm{~km} / \mathrm{mol})$ that is certainly unphysical, while the upper band, observed in accurate studies to be of the same order of magnitude as the lower one, is found here to be almost two orders of magnitude less intense. We can say with some certainty that these intensities are not realistic.

Going to a CASSCF (4/4) calculation very significantly affects the frequencies of the two highest $\sigma_{\mathrm{u}}$ bands, but leaves the other stretches virtually untouched. However, the intensity problem is not resolved, while the third IR band becomes almost invisible by comparison to the two others. At the CASSCF (8/8) level, the two uppermost $\sigma_{\mathrm{g}}$ modes become affected (especially the top one), and, to a lesser extent, the third $\sigma_{\mathfrak{u}}$ mode. The intensity problem appears to be less acute, in that now at least all hands have physically plausible intensities. Examination of natural orbital occupation numbers suggests that the CASSCF $(8 / 8)$ treatment accounts for most of the nondynamical correlation effects, and any further expansion of the active space would really correspond to describing dynamical correlation, for which CASSCF is not an effective treatment. Increasing the active space further in any balanced way would also probably require inclusion of $\sigma$ orbitals, which would anyway lead to calculations too large to be feasible. Thus CASSCF (8/8) seems to be an appropriate and economic level of treatment.

We can now turn to $\mathrm{C}_{13}$. The HF/cc-pVDZ frequencies are dominated by an anomalously intense band at $1720 \mathrm{~cm}^{-1}$. Going to $\operatorname{CASSCF}(8 / 8)$ drastically changes not only the frequencies of the third and fourth highest $\sigma_{\mathrm{u}}$ bands, but also the intensity behavior. Now we have extremely intense bands calculated at 1843 and $2003 \mathrm{~cm}^{-1}$; with more account for external correlation, either band could come close enough 
Table 2

Computed frequencies $\left(\mathrm{cm}^{-1}\right)$ and infrared intensities $(\mathrm{km} / \mathrm{mol})$ for linear $\mathrm{C}_{13}$

\begin{tabular}{llrlr}
\hline & \multicolumn{3}{l}{ SCF/cc-pVDZ } & \multicolumn{2}{c}{ CASSCF $(8 / 8) / c c-p V D Z$} \\
\hline$\sigma_{\mathrm{u}}$ & 2419 & 703 & 2409 & 136 \\
$\sigma_{\mathrm{g}}$ & 2390 & 0 & 2346 & 0 \\
$\sigma_{\mathrm{g}}$ & 2333 & 0 & 2318 & 0 \\
$\sigma_{\mathrm{u}}$ & 2245 & 72 & 2227 & 200 \\
$\sigma_{\mathrm{g}}$ & 2099 & 0 & 2083 & 0 \\
$\sigma_{\mathrm{u}}$ & 1874 & 3616 & 2003 & 84067 \\
$\sigma_{\mathrm{u}}$ & 1720 & $>10^{5}$ & 1843 & 13738 \\
$\sigma_{\mathrm{g}}$ & 1587 & 0 & 1573 & 0 \\
$\sigma_{\mathrm{u}}$ & 1300 & 1 & 1294 & 88 \\
$\sigma_{\mathrm{g}}$ & 996 & 0 & 987 & 0 \\
$\pi_{\mathrm{u}}$ & 804 & 71 & 793 & 46 \\
$\pi_{\mathrm{g}}$ & 725 & 0 & 722 & 0 \\
$\sigma_{\mathrm{u}}$ & 677 & 253 & 678 & 69 \\
$\pi_{\mathrm{u}}$ & 656 & 6 & 649 & 9 \\
$\pi_{\mathrm{g}}$ & 591 & 0 & 587 & 0 \\
$\pi_{\mathrm{u}}$ & 534 & 0 & 511 & 0 \\
$\sigma_{\mathrm{g}}$ & 340 & 0 & 341 & 0 \\
$\pi_{\mathrm{g}}$ & 275 & 0 & 295 & 0 \\
$\pi_{\mathrm{u}}$ & 239 & 2 & 244 & 2 \\
$\pi_{\mathrm{g}}$ & 183 & 0 & 192 & 0 \\
$\pi_{\mathrm{u}}$ & 119 & 4 & 124 & 3 \\
$\pi_{\mathrm{g}}$ & 65 & 0 & 68 & 0 \\
$\pi_{\mathrm{u}}$ & 25 & 4 & 27 & 5 \\
\hline & & & & \\
\hline
\end{tabular}

to $1809 \mathrm{~cm}^{-1}$ to correspond to the feature seen by GOHFPS. Hence we are clearly dealing with a situation here where nondynamical correlation effects essentially preclude any useful prediction at the SCF level, as well as (of course) from low-order perturbation theory. The anomalously high intensities still suggest that an even higher-level calculation would be desirable, however, it is hard to see what more sophisticated calculation would be technically feasible at present.

Having discussed $C_{9}$ and $C_{13}$, we may look at results for $\mathrm{C}_{11}$. These are given in Table 3 . Interestingly, here intensity patterns of the SCF and $\operatorname{CASSCF}(8 / 8)$ frequencies are quite similar, while the effect on the actual frequencies is also relatively small (the second through fourth $\sigma_{\mathrm{g}}$ and $\sigma_{\mathrm{u}}$ modes, counting from the top, are affected significantly). Still, we see one band with an anomalously high intensity, which might take on a somewhat more physically plausible value if the active space were enlarged (which is presently beyond the realm of the computationally feasible). Qualitatively, however, the computed spectrum is in agreement with the $\mathrm{HF} / 6-31 \mathrm{G}^{*}$ values presented previously
Table 3

Computed frequencies $\left(\mathrm{cm}^{-1}\right)$ and infrared intensities $(\mathrm{km} / \mathrm{mol})$ for linear $\mathrm{C}_{11}$

\begin{tabular}{lrrrr}
\hline & \multicolumn{2}{c}{ SCF/cc-pVDZ } & \multicolumn{2}{c}{ CASSCF $(8 / 8) / c c-p V D Z$} \\
\hline$\sigma_{\mathrm{g}}$ & 2408 & 0 & 2407 & 0 \\
$\sigma_{\mathrm{u}}$ & 2400 & 80 & 2387 & 1 \\
$\sigma_{\mathrm{g}}$ & 2269 & 0 & 2236 & 0 \\
$\sigma_{\mathrm{u}}$ & 2126 & 2400 & 2075 & 1378 \\
$\sigma_{\mathrm{u}}$ & 1913 & 81231 & 1895 & 93266 \\
$\sigma_{\mathrm{g}}$ & 1842 & 0 & 1804 & 0 \\
$\sigma_{\mathrm{u}}$ & 1507 & 147 & 1478 & 130 \\
$\sigma_{\mathrm{g}}$ & 1162 & 0 & 1144 & 0 \\
$\sigma_{\mathrm{u}}$ & 795 & 107 & 787 & 253 \\
$\pi_{\mathrm{u}}$ & 771 & 65 & 779 & 60 \\
$\pi_{\mathrm{g}}$ & 694 & 0 & 697 & 0 \\
$\pi_{\mathrm{u}}$ & 617 & 3 & 620 & 0 \\
$\pi_{\mathrm{g}}$ & 547 & 0 & 550 & 0 \\
$\sigma_{\mathrm{g}}$ & 406 & 0 & 406 & 0 \\
$\pi_{\mathrm{u}}$ & 269 & 1 & 276 & 7 \\
$\pi_{\mathrm{g}}$ & 226 & 0 & 241 & 0 \\
$\pi_{\mathrm{u}}$ & 156 & 4 & 162 & 9 \\
$\pi_{\mathrm{g}}$ & 87 & 0 & 87 & 0 \\
$\pi_{\mathrm{u}}$ & 34 & 4 & 34 & 6 \\
\hline
\end{tabular}

by Martin et al. [34].

It should be remarked that in all cases, the APT population analysis [35], which is based on the dipole gradient tensor, produces populations which are qualitatively at odds with conventional expectations (such as carbon atom populations exceeding \pm 4 ). This is indicative of very large charge movement along the molecular axis and a rapidly changing (with geometry) ionic contribution to the wave function. Also noteworthy are the very large polarizabilities along the molecular axis: $131.5 \AA^{3}$ for $C_{11}, 172.5 \AA^{3}$ for $C_{13}$. All these results suggest that carbon fiber materials could have interesting nonlinear optical properties.

\section{Conclusions}

We are in a position to state that:

- as the length of linear carbon clusters $C_{2 n+1}$, and especially $\mathrm{C}_{4 n+1}$, increases, nondynamical correlation effects on the harmonic frequencies and intensities become noticeable;

- at the CASSCF (8/8)/cc-pVDZ level a band corresponding to the observed transition for $\mathrm{C}_{13}$ is found, with a very high computed intensity; 
- for $\mathrm{C}_{9}$, at least a $\mathrm{CASSCF}(4 / 4)$ is required to reproduce the ordering of the frequencies correctly;

- the computed infrared intensities change qualitatively with the size of the active space.

\section{Acknowledgement}

JMLM thanks the National Science Foundation of Belgium (NFWO/FNRS) for a Postdoctoral Fellowship. Part of this work was supported by a grant of computer time from the San Diego Supercomputer Center. PRT was supported by the National Science Foundation under Cooperative Agreement DASC8902825 and Grant CHE-9320718. The authors finally wish to thank Profesor R.J. Saykally for helpful discussions.

\section{References}

[1] W. Weltner Jr. and R.J. Van Zee, Chem. Rev. 89 (1989) 1713.

[2] J.M.L. Martin, J.P. Francois, and R. Gijbels, J. Mol. Struct. 294 (1993) 21.

[3] J. Hutter, H.P. Lïthi, and F. Diederich, J. Am. Chem. Soc. 116 (1994) 750.

[4] K.P. Huber and G. Herzberg, Constants of diatomic molecules (Van Nostrand Reinhold, New York, 1979).

[5] J.M.L. Martin and P.R. Taylor, submitted for publication.

[6] K.R. Thompson, R.L. DeKock and W. Weltner Jr., J. Am. Chem. Soc. 93 (1971) 4688.

17] W. Krätschmer and K. Nachtigall, in: Polycyclic aromatic hydrocarbons and astrophysics, ed. A. Léger et al. (Reidel, Dordrecht, 1987) p. 75;

K. Nachtigall, Diplomarbeit (University of Heidelberg, Germany, 1987).

[8] J.M.L. Martin, J.P. Francois and R. Gijbels, J. Chem. Phys. 94 (1991) 3753.

[9] J.M.L. Martin, P.R. Taylor, T.J. Lee and D.W. Schwenke, submitted for publication.

[10] J.M.L. Martin and P.R. Taylor, J. Chem. Phys., in press.

[11] T.F. Giesen, A. Vanorden, H.J. Hwang, R.S. Fellers, R.A. Provencal and R.J. Saykally, Science 265 (1994) 756.
[12] J.R. Heath and R.J. Saykally, in: On clustcrs and clustering, ed. P.J. Reynolds (Elsevier, Amsterdam, 1993) pp. 7-21, and references therein.

[13] G. Von Helden, N.G. Gotts and M.T. Bowers, Chem. Phys. Letters 212 (1993) 241.

[14] G. Von Helden, W.E. Palke and M.T. Bowers, Chem. Phys. Letters 212 (1993) 247.

[15] J.M.L. Martin, J.P. Francois and R. Gijbels, J. Chem. Phys. 93 (1990) 8850.

[16] L.N.J.R. Heath and R.J. Saykally, J. Chem. Phys. 94 (1991) 3271 , and references therein.

[17] N. Moazzen-Ahmadi, A.R.W. McKellar and T. Amano, J. Chem. Phys. 91 (1989) 2140, and references therein.

[18] H.J. Hwang, A. Vanorden, K. Tanaka, E.W. Kuo, J.R. Heath and R.J. Saykally, Mol. Phys. 79 (1993) 769, and references therein.

[19] R.H. Kranze and W.R.M. Graham, J. Chem. Phys. 98 (1993) 71.

[20] J.R. Heath, A. Vanorden, E. Kuo and R.J. Saykally, Chem. Phys. Letters 182 (1991) 17, and references therein.

[21] J.R. Heath and R.J. Saykally, J. Chem. Phys. 93 (1990) 8392.

[22] J.M.L. Martin, unpublished calculations.

[23] L. Adamowicz and J. Kurtz, Astrophys. J. 370 (1991) 784.

[24] T.H. Dunning Jr., J. Chem. Phys. 90 (1989) 1007.

[25] J.M.L. Martin and T.J. Lee, J. Chem. Phys. 98 ( 1993) 795 I.

[26] J.M.L. Martin, T.J. Lee and P.R. Taylor, submitted for publication

[27] S. Huzinaga, J. Chem. Phys. 42 (1965) 1293.

[28] T.H. Dunning Jr., J. Chem. Phys. 53 (1970) 2823.

[29] B.O. Roos, Advan. Chem. Phys. 69 (1987) 399.

[30] H.J.Aa. Jensen, H. Ågren and J. Olsen, SIRIUS, a multiconfigurational SCF program;

T. Helgaker, K.L. Bak, H.J.Aa. Jensen, P. Jørgensen, R. Kobayashi, H. Koch, K. Mikkelsen, J. Olsen, K. Ruud, P.R. Taylor and $O$. Vahtras, ABACUS, a second-order MCSCF molecular properties program.

[31] R. Ahlrichs, M. Bär, M. Häser, H. Horn and C. Kölmel, Chem. Phys. Letters 162 (1989) 165.

[32] J.M.L. Martin, J.P. François and R. Gijbels, J. Comput. Chem. 12 (1991) 52.

[33] M.J.S. Dewar and W. Thiel, J. Am. Chem. Soc. 99 (1977) 4899.

[34] J.M.L. Martin, J.P. François, R. Gijbels and J. Almlöf, Chem. Phys. Letters 187 (1991) 367.

[35] J. Cioslowski, J. Am. Chem. Soc. 111 (1989) 8333. 